\title{
Efeitos da fotobiomodulação (laser de baixa intensidade) na cicatrização de feridas: revisão integrativa
}

\author{
Effects of photobiomodulation (low-intensity laser) on wound healing: an integrative review \\ Efectos de la fotobiomodulación (láser de baja intensidad) en la cicatrización de heridas: una \\ revisión integradora
}

Recebido: 24/08/2021 | Revisado: 29/08/2021 | Aceito: 31/08/2021 | Publicado: 04/09/2021

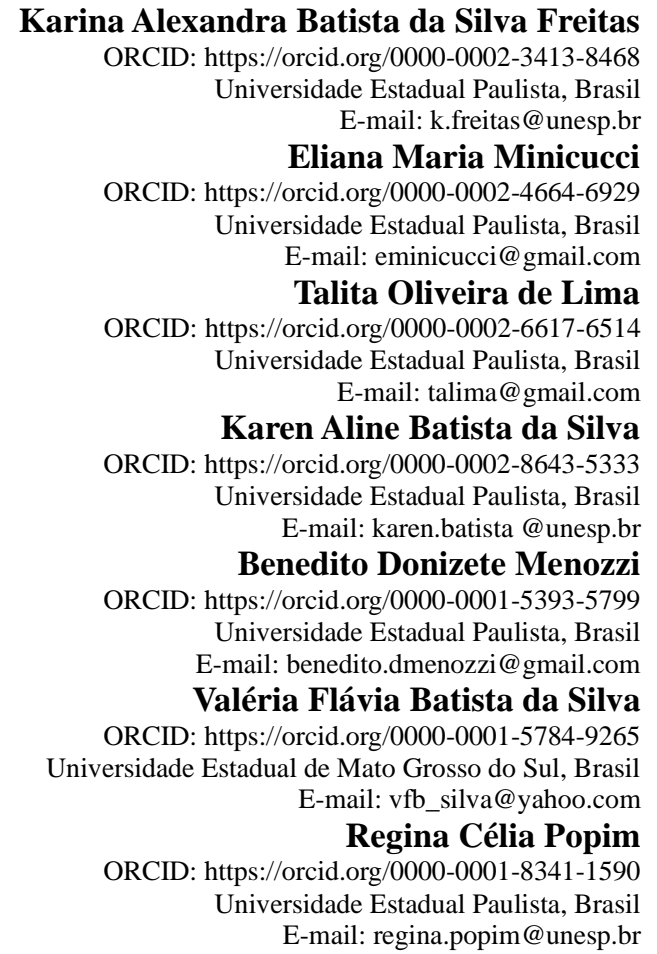

\begin{abstract}
Resumo
Objetivos: Identificar e analisar as evidências existentes referentes ao uso da terapia com luz de baixa intensidade na cicatrização de feridas. Método: Revisão integrativa baseada no referencial de Ganong, com busca realizada em março de 2020, com recorte temporal de janeiro de 2015 a março de 2020. Resultados: Foram selecionados 15 estudos, com diferentes delineamentos de pesquisa. Observou-se uma diferença nos parâmetros utilizados com energias que variaram de 1 a $20 \mathrm{~J}$, o comprimento de onda entre 632,8 e $940 \mathrm{~nm}$ e potência de 1,08 mW a 6W. Conclusões: Não há protocolo clínico estabelecido para a cicatrização de feridas com uso da terapia com luz de baixa intensidade, observase que há efeitos benéficos da fotobiomodulação com adequados comprimento de onda, densidade de energia e potência do aparelho. Há necessidade de novos estudos com finalidade de melhor entendimento dessa terapia e estabelecimento de protocolos clínicos na prática em saúde.

Palavras-chave: Fotobiomodulação; Cuidados de enfermagem; Cicatrização de feridas; Protocolos clínicos; Terapia a laser.

\footnotetext{
Abstract

Objectives: To identify and analyze the existing evidence regarding the use of low-intensity light therapy in wound healing. Method: Integrative review based on the Ganong framework, with a search conducted in March 2020, with a period from January 2015 to March 2020. Results: 15 studies were selected, with different research designs. There was a difference in the parameters used with energies ranging from 1 to $20 \mathrm{~J}$, the wavelength between 632.8 and 940 $\mathrm{nm}$ and power from $1.08 \mathrm{~mW}$ to $6 \mathrm{~W}$. Conclusions: There is no established clinical protocol for wound healing using low intensity light therapy; it is observed that there are beneficial effects of photobiomodulation with adequate wavelength, energy density and power of the device. Further studies are needed to better understand this therapy and establish clinical protocols in health practice.
} 
Keywords: Photobiomodulation; Nursing care; Wound healing; Clinical protocols; Laser therapy.

\section{Resumen}

Objetivos: Identificar y analizar la evidencia existente sobre el uso de la fototerapia de baja intensidad en la cicatrización de heridas. Método: Revisión integrativa basada en el marco de Ganong, con búsqueda realizada en marzo de 2020, con un marco temporal de enero de 2015 a marzo de 2020. Resultados: Se seleccionaron 15 estudios, con diferentes diseños de investigación. Hubo una diferencia en los parámetros utilizados con energías que van de 1 a $20 \mathrm{~J}$, la longitud de onda entre 632,8 y $940 \mathrm{~nm}$ y la potencia de $1,08 \mathrm{~mW}$ a $6 \mathrm{~W}$. Conclusiones: No existe un protocolo clínico establecido para la cicatrización de heridas mediante fototerapia de baja intensidad, se observa que existen efectos beneficiosos de la fotobiomodulación con adecuada longitud de onda, densidad de energía y potencia del dispositivo. Se necesitan más estudios para comprender mejor esta terapia y establecer protocolos clínicos en la práctica de la salud.

Palabras clave: Fotobiomodulación; Cuidado de enfermera; Cicatrización de la herida; Protocolos clínicos; Terapia con láser.

\section{Introdução}

A pele é o maior órgão do corpo humano sendo a primeira barreira de proteção contra agentes externos, perda de água, invasão de microrganismos. É composta por duas camadas distintas: a epiderme e a derme. Logo abaixo da derme encontra-se a hipoderme que é constituída de um tecido subcutâneo adiposo (Szwed \& Santos, 2016; Oliveira \& Dias, 2012).

A ferida é uma interrupção da continuidade da pele, podendo ser de diversas etiologias: traumática, isquemia, infecção, cirúrgica, por pressão, de tamanho e caraterísticas variáveis. Quando ocorre um rompimento tecidual inicia-se um processo de reparo complexo com objetivo de restauração do tecido (Oliveira et al., 2012).

Não existem dados concretos sobre a prevalência de feridas na população brasileira, porém existem estudos que estimam a prevalência de indivíduos com tal lesão em 1,05\% na população em geral e de 1,9\% entre aqueles atendidos na atenção primária à saúde (Lima et al., 2018).

Após uma lesão, ocorre uma cascata perfeita de eventos moleculares complexos intercedidos e sustentados por um conjunto de mediadores químicos agindo em diferentes fases, com objetivo de realizar a cicatrização da ferida. As fases possuem limites não muito distintos, mas sobrepostas no tempo, denominadas: fase inflamatória, proliferativa e de remodelação (Oliveira et al., 2012; Beheregaray et al., 2017; Marteli et al., 2018).

A fase inflamatória tem duração de 48 a 96 h após o ferimento ocorrendo hemostasia por meio da ativação da cascata de coagulação, formando o tampão de plaquetas. Há o aumento da permeabilidade vascular com o recrutamento de neutrófilos e macrófagos evidenciando os sinais físicos do processo inflamatório: eritema, edema, calor e dor. Inicia-se a cascata de reparação por meio do apoio, recrutamento e ativação celular, síntese da matriz extracelular e o início do processo da angiogênese. Na fase proliferativa os eventos da inflamação promovem a limpeza da ferida por meio da fagocitose. Ocorre de 4 a 12 dias após a lesão e algumas células começam a realizar a epitelização da lesão como os fibroblastos, células musculares lisas, células endoteliais e epiteliais. Já a fase de remodelação é o último e mais longo processo de cicatrização podendo ser continuado por 12 a 18 meses. A principal característica dessa fase é a presença do colágeno, principal componente da derme. A matriz de colágeno possui aproximadamente $20 \%$ de colágeno tipo III, o que a torna extremamente frágil. A mudança para colágeno tipo I é gradual sendo aumentada pelos fibroblastos (Szwed et al., 2016; Marteli et al., 2018, Medeiros et al., 2016).

Fatores locais e sistêmicos podem ser responsáveis pela diminuição no processo de cicatrização, aumentando o tempo da lesão, tais como: tamanho e localização da ferida, tipo de tecido presente (necrose, esfacelo), contaminação bacteriana, presença de biofilme, técnica incorreta na realização do curativo, diabetes, doenças auto - imunes, patologias vasculares, uso de corticosteroides, tratamento oncológico (quimioterapia e /ou radioterapia) e desnutrição. Esses fatores causam um atraso na formação do tecido de granulação, proliferação de fibroblastos e neovascularização (Szwed et al., 2016; Oliveira et al., 2012; Medeiros et al., 2016). 
A enfermagem demonstra um papel fundamental na evolução de coberturas, proporcionando melhorias no tratamento e consequente avanço tecnológico, garantindo a otimização de materiais e a qualidade na assistência. É fundamental que o enfermeiro compreenda toda complexidade da cicatrização de feridas, pois a escolha inadequada de um produto pode comprometer todo o processo de cura da ferida (Lima et al., 2018; Vieira et al., 2017).

Nesse sentido, novas tecnologias devem ser incorporadas na cicatrização de feridas, o enfermeiro deve buscar conhecimentos acerca de novas técnicas sobre a regeneração da pele e aceleração do processo cicatricial (Lima et al., 2018).

Atualmente a fotobiomodulação (FBM) é utilizada para acelerar o processo cicatricial e em diversas aplicações médicas, promovendo melhorias na qualidade de vida dos pacientes e otimizando o tratamento (Silva Neto et al., 2017; Gomes \& Schapochnick, 2017; Chung et al., 2012). É importante alternativa no tratamento de processos cicatriciais, pois possuem ações anti-inflamatórias, analgésicas e de cicatrização. Além disso, não causam efeitos colaterais como aqueles induzidos por alguns fármacos (por exemplo, os corticoides), evidenciando uma melhora do prognóstico do paciente (Silva Neto et al., 2017; Gomes et al., 2017; Chamusca et al. 2012).

Para a aplicação da FBM, devem ser considerados parâmetros como comprimento de onda, fluência, densidade de potência e tempo da luz aplicada. A escolha do parâmetro dependerá da especificidade do tratamento, portanto, uma escolha errada desses parâmetros pode significar em eficácia reduzida, ou mesmo em um resultado terapêutico negativo. A FBM é caracterizada por uma dose-resposta bifásica, onde doses mais baixas de luz são frequentemente mais benéficas que doses altas (Chung et al., 2012).

Infere-se que, apesar de a FBM ser uma possibilidade tecnológica que pode contribuir no processo de cicatrização, existem dúvidas quanto aos parâmetros empregados, justificando esse estudo.

Dessa forma, objetivamos com esse estudo identificar e analisar as evidências existentes referentes ao uso da terapia com luz de baixa intensidade na cicatrização de feridas.

\section{Metodologia}

Trata-se de uma pesquisa de revisão integrativa da literatura referente à produção científica da FBM no tratamento de feridas.

A revisão integrativa proporciona a síntese do conhecimento por meio de um tema ou questão delimitada de maneira sistemática e ordenada. Auxilia na tomada de decisões, aponta lacunas do conhecimento promovendo pensamento crítico sugerindo novos estudos. Foi utilizado Ganong (1987) como referencial teórico, considerando-se as etapas: elaboração da questão norteadora; busca da literatura, coleta de dados, análise crítica dos resultados, discussão dos resultados e relato da revisão (Souza et al., 2010).

A questão norteadora foi realizada considerando-se a estratégia PICO (Santos et al., 2007) e foi a seguinte: A FBM favorece a cicatrização de feridas de diversas etiologias? Quais os parâmetros indicados para cicatrização? Desta forma, considerou-se P (paciente)= humanos/animais com feridas; I (Intervenção)= Uso de fotobiomodulação (vermelho e infra vermelho); $\mathrm{C}$ (comparação)= grupos que não realizaram laser de baixa intensidade; $\mathrm{O}($ desfecho $)=$ favorecimento da cicatrização.

Os critérios de inclusão foram: pesquisas que abordassem o tema de FBM em feridas (qualquer tipo de ferida), em formato de artigos com textos disponíveis, nos idiomas português, inglês e espanhol, que estivessem indexados e artigos que retratassem o uso do laser de Diodo. O recorte temporal foi de 5 anos e 3 meses (janeiro de 2015 a março de 2020), para garantir uma pesquisa recente, já que a FBM é utilizada já há algumas décadas.

Foram considerados como critério de exclusão: publicações fora do período estabelecido, artigos que não retratassem especificamente o uso da FBM, pesquisas relacionadas à odontologia, pois já se sabe o benefício da mesma nessa especialidade 
e artigos que retratassem o uso de do laser de HeNe. A busca foi realizada no mês de março de 2020, nas fontes eletrônicas: Biblioteca Virtual de Saúde (BVS), U.S. National Library os Medicine (Portal PubMed), Scientific Electronic Library Online (SCIELO). A estratégia de busca utilizou os descritores e seus sinônimos. Foi respeitado o método que cada base da dados exigia, utilizando-se os operadores boleanos AND e OR para garantir a busca ampla. Foram utilizados os descritores: "Terapia com luz de baixa intensidade", "Low-Level Light Therapy", "Terapia por Luz de Baja Intensidad" e "Cicatrização de feridas", "Wound Healing", "Cicatrización de Heridas" bem como os seus sinônimos, todos registrados nos Descritos em Ciências da Saúde (DeCS) e Medical Subjecti Headings (MeSH).

Além disso, para mostrar as etapas de seleção dos artigos, usou-se o Check List Preferred Items for Systematic Reviews and Meta-Analyses (PRISMA) (Moher et al., 2009) (Figura1).

Figura 1 - Fluxograma do Cruzamento e resultados da busca, segundo recomendação de Check List Preferred Items for Systematic Reviews and Meta-Analyses, 2020.

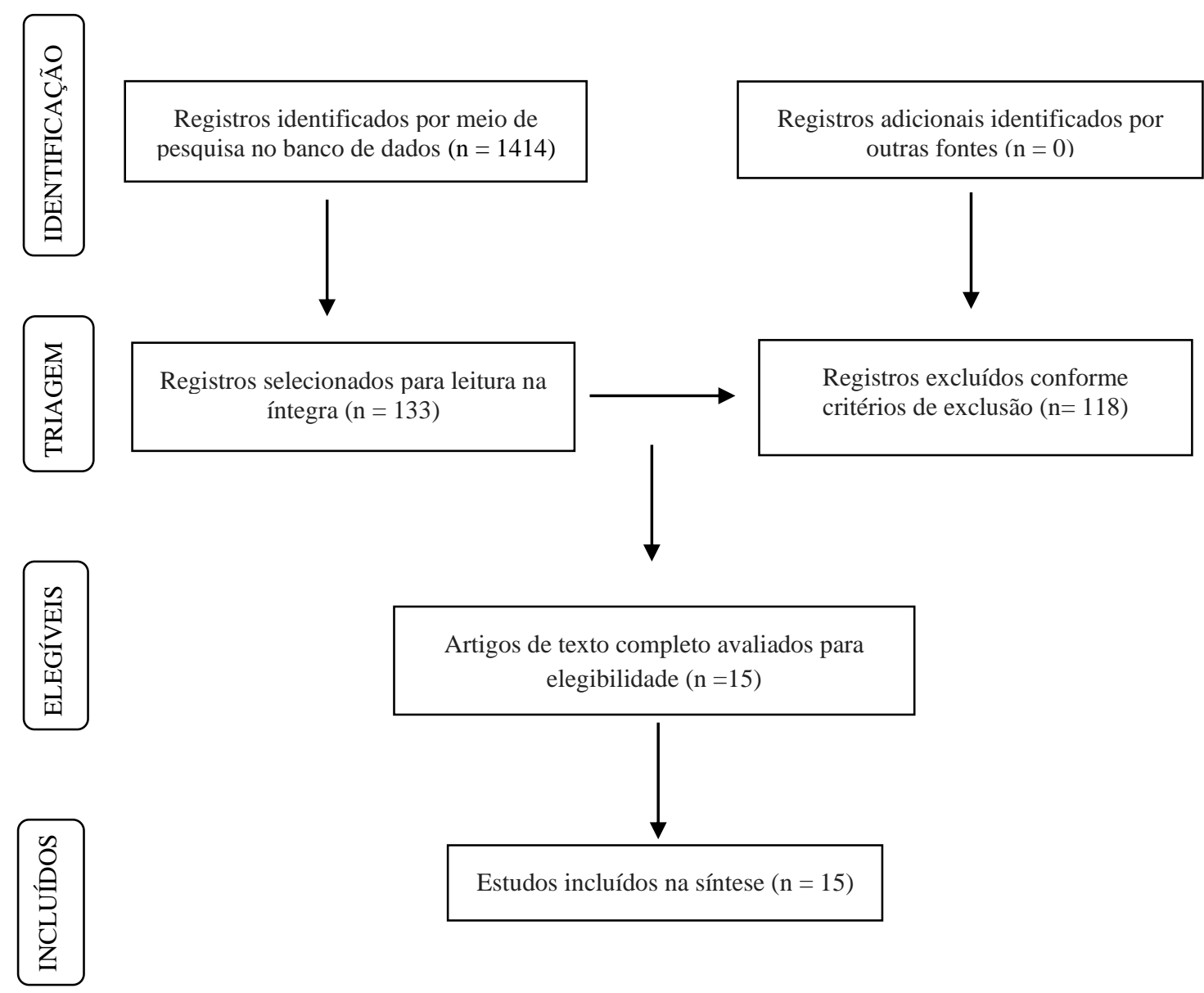

Fonte: Autores (2021).

A busca encontrou 1414 publicações e após aplicação dos critérios de inclusão, elegibilidade e exclusão, selecionouse 15 artigos para análise.

Após a seleção dos artigos, para a coleta de dados, utilizou-se um instrumento validado (Ursi \&Galvão, 2006), voltado para a revisão integrativa abordando: autor/referência; ano/país; delineamento/número de pacientes; intervenções e desfecho. Além disso, adaptou-se à pesquisa para responder a pergunta norteadora, os critérios: quantidade de joules utilizados, 
comprimento de onda, frequência de aplicação, nível de evidência. Ressaltamos que algumas questões foram suprimidas durante sua aplicação.

Os artigos também foram classificados de acordo com a qualidade da evidência em seis níveis (Souza et al., 2010):

Nível 1 - evidências que resultam da metanálise de múltiplos estudos clínicos controlados e randomizados;

Nível 2 - evidências por meio de estudo individual com delineamento experimental;

Nível 3 - estudo com delineamento quase experimental;

Nível 4 - estudos descritivos, (não experimental) ou com abordagem qualitativa;

Nível 5 - relatório de casos ou de experiência;

Nível 6 - opinião de especialistas.

\section{Resultados e Discussão}

Foram encontrados 1414 estudos. Do total, 1368 foram localizados na PUBMED, 17 na BVS e 29 na SCIELO, sendo que após análise, seguindo os critérios de inclusão/exclusão, selecionou-se 15 artigos científicos que compuseram a revisão. Foram provenientes da PubMed 7 artigos, 07 da BVS e 01 da SCIELO (Tabela 1).

Em 2017, houve um crescimento na quantidade de artigos publicados, apresentando cinco publicações $(31,25 \%)$. Os estudos apresentaram diferentes delineamento da pesquisa e consequentemente diferentes níveis de evidência. $\mathrm{O}$ estudo mais comum foi o experimental com onze artigos (73,3\%), três ensaios clínicos (20\%) e uma série de casos $(6,6 \%)$. Dez estudos $(66,6 \%)$ foram realizados no Brasil, os demais no Irã, Polônia e Turquia. Com relação ao nível de evidência, 73,3\% apresentaram nível 2, 13,3\% nível 3, 6,6\% nível 1 e 6,6\% nível 4.

Quadro 1 - Caracterização dos artigos analisados de acordo com autor, referência, ano/país, delineamento/número de pacientes, intervenções e desfecho, Botucatu, São Paulo, Brasil, 2020.

\begin{tabular}{|c|c|c|c|c|}
\hline $\begin{array}{l}\text { Autor/ } \\
\text { Ano }\end{array}$ & País & $\begin{array}{c}\text { Delineamento/Nú } \\
\text { mero de } \\
\text { Pacientes }\end{array}$ & Intervenções & Desfecho \\
\hline $\begin{array}{l}\text { Barbosa, et al. } \\
\quad(2020)\end{array}$ & Brasil & $\begin{array}{c}\text { Estudo } \\
\text { experimental } \\
\mathrm{n}=24 \text { ratos Wistar } \\
\text { machos }\end{array}$ & $\begin{array}{l}\text { Realizado dissecação da pele até exposição da } \\
\text { fáscia muscular com área de } 2 \mathrm{~cm}^{2} \text {, após os } \\
\text { animais foram randomizados em } 4 \text { grupos com } \\
\text { objetivo de investigar os efeitos da } \\
\text { fotobiomodulação com comprimento de onda de } \\
658,830 \text { e } 904 \mathrm{~nm} \text { com potência de } 30 \mathrm{~mW} \text { no } \\
\text { reparo de lesões cutâneas em modelo animal } \\
4 \mathrm{~J} / \mathrm{cm}^{2} \text { - diariamente por } 14 \text { dias }\end{array}$ & $\begin{array}{l}\text { O comprimento de onda } 658 \mathrm{~nm} \\
\text { foi mais eficaz para estimular o } \\
\text { reparo do tecido. }\end{array}$ \\
\hline $\begin{array}{c}\text { Scherer, et al. } \\
\text { (2019) }\end{array}$ & Brasil & $\begin{array}{c}\text { Estudo } \\
\text { experimental } \\
\mathrm{n}=24 \text { ratos Wistar } \\
\text { machos }\end{array}$ & $\begin{array}{l}\text { Os animais foram expostos a fumaça do cigarro } \\
\text { comercial, após, os animais que não } \\
\text { participaram do grupo controle foram } \\
\text { submetidos a dano do tendão calcâneo para } \\
\text { simular os tratamentos. O protocolo de } \\
\text { fotobiomodulação utilizado foi de: } 3 \mathrm{~J} / \mathrm{cm}^{2} \text {, } \\
\text { comprimento de onda de } 830 \mathrm{~nm} \text { e potência de } \\
30 \mathrm{~mW} \text {. }\end{array}$ & $\begin{array}{l}\text { Após aplicação do laser na lesão } \\
\text { do tendão do calcâneo, houve } \\
\text { diminuição na atividade da } \\
\text { enzima mieloperoxidase } \\
\text { diminuindo assim, a resposta } \\
\text { inflamatória aguda. }\end{array}$ \\
\hline Filho, et al. (2017) & Brasil & $\begin{array}{l}\quad \text { Estudo } \\
\text { experimental, } \\
\mathrm{n}=42 \text { coelhos } \\
\quad \text { adultos }\end{array}$ & $\begin{array}{l}\text { Foi realizado um enxerto de pele e de membrana } \\
\text { amniótica de medidas } 2 \mathrm{~cm} \times 2 \mathrm{~cm} \text {, do dorso do } \\
\text { animal. Os animais do grupo laser foram } \\
\text { submetidos a dosagem de } 4 \mathrm{~J} / \mathrm{cm}^{2} \text { no } \\
\text { comprimento de onda de } 660 \mathrm{~nm} \text { e potência de } \\
30 \mathrm{~mW} \text {. }\end{array}$ & $\begin{array}{l}\text { Quando comparado aos outros } \\
\text { grupos, o laser apresentou } \\
\text { melhores respostas, mostrando } \\
\text { um processo cicatricial mais } \\
\text { eficiente promovendo uma } \\
\text { melhor integração do enxerto. }\end{array}$ \\
\hline
\end{tabular}




\begin{tabular}{|c|c|c|c|c|}
\hline Atasoy, et al. (2017) & Turquia & $\begin{array}{c}\text { Estudo } \\
\text { experimental, } \\
\mathrm{n}=48 \text { ratos } \\
\text { Wistar fêmeas }\end{array}$ & $\begin{array}{l}\text { Foi realizado um defeito ósseo na tíbia dos } \\
\text { animais de } 5 \text { x } 2 \mathrm{~mm} \text {, após foi realizado a } \\
\text { laserterapia de baixa intensidade nos parâmetros: } \\
\text { comprimento de onda de } 940 \mathrm{~nm} \text { com } \\
\text { intensidades de energia diferentes }(5,10 \text {, } \\
\left.20 \mathrm{~J} / \mathrm{cm}^{2}\right) \text { e potências diferentes }(1,5 ; 3,6 \mathrm{~W}) \mathrm{em} \\
\text { dias alternados }\end{array}$ & $\begin{array}{l}\text { Não foram encontradas } \\
\text { diferenças entre as densidades de } \\
\text { energia durante a análise } \\
\text { histológica. A aplicação do laser } \\
\text { de baixa intensidade e } 940 \mathrm{~nm} \\
\text { pode não acelerar o processo de } \\
\text { regeneração óssea. }\end{array}$ \\
\hline $\begin{array}{l}\text { Carvalho et al. } \\
\text { (2016) }\end{array}$ & Brasil & $\begin{array}{l}\text { Estudo de Caso } \\
\text { Clínico, } \mathrm{n}=32 \\
\text { pacientes }\end{array}$ & $\begin{array}{l}\text { Foi realizado a laserterapia de baixa intensidade } \\
\text { isolada e associada ao óleo de calêndula } \\
\text { officinalis no reparo de úlceras em pé diabéticos, } \\
\text { utilizando-se a dose de } 4 \mathrm{~J} / \mathrm{cm}^{2} \text {, com } \\
\text { comprimento de onda de } 658 \mathrm{~nm} \text { e potência de } \\
30 \mathrm{~mW}, 3 x \text { na semana. }\end{array}$ & $\begin{array}{l}\text { A laserterapia isolada ou } \\
\text { associada ao óleo de Calêndula } \\
\text { officinalis foi eficaz no alívio da } \\
\text { dor e na redução da área da } \\
\text { ferida. }\end{array}$ \\
\hline Sousa, et al. (2015) & Brasil & $\begin{array}{l}\text { Estudo } \\
\text { Experimental, } \mathrm{n}= \\
40 \text { ratos machos } \\
\text { família Muridae }\end{array}$ & $\begin{array}{l}\text { Realizado grupos comparando o uso da } \\
\text { laserterapia em diferentes densidades de energia } \\
\left(5 \mathrm{e} 8 \mathrm{~J} / \mathrm{cm}^{2} \text { ) diariamente, com comprimento de }\right. \\
\text { onda de } 660 \mathrm{~nm} \text { e potência de } 30 \mathrm{mw} \text {.com o } \\
\text { gerador de alta frequência para avaliar o } \\
\text { processo de cicatrização de feridas em ratos. }\end{array}$ & $\begin{array}{l}\text { A laserterapia com a densidade } \\
\text { de energia de } 5 \mathrm{~J} / \mathrm{cm}^{2} \text { apresentou } \\
\text { mais eficácia na fase aguda do } \\
\text { processo de cicatrização. Na fase } \\
\text { de remodelação ambas } \\
\text { apresentaram resultados } \\
\text { relevantes. A densidade de } \\
8 \mathrm{~J} / \mathrm{cm}^{2} \text { apresentou melhores } \\
\text { resultados aos } 14 \text { dias. O gerador } \\
\text { de alta frequência, não } \\
\text { apresentou resultados } \\
\text { satisfatórios. }\end{array}$ \\
\hline Feitosa, et al. (2015) & Brasil & $\begin{array}{c}\text { Ensaio Clínico } \\
\text { Randomizado, } \mathrm{n}= \\
16 \text { pacientes }\end{array}$ & $\begin{array}{l}\text { Foi aplicado a laserterapia de baixa intensidade } \\
\text { com dose de } 4 \mathrm{~J} / \mathrm{cm}^{2}, 3 x \text { na semana, em dias } \\
\text { alternados com comprimento de onda de } \\
632,8 \mathrm{~nm} \text {, com potência de } 30 \mathrm{~mW} \text {, em pacientes } \\
\text { portadores de úlceras diabéticas, para avaliar os } \\
\text { efeitos dessa terapêutica no reparo tecidual. }\end{array}$ & $\begin{array}{l}\text { O laser de baixa intensidade } \\
\text { apresentou eficiência, } \\
\text { melhorando significativamente a } \\
\text { dor do paciente. }\end{array}$ \\
\hline $\begin{array}{l}\text { Martins, et al. } \\
\qquad(2015)\end{array}$ & Brasil & $\begin{array}{c}\text { Estudo } \\
\text { Experimental, } \mathrm{n}= \\
40 \text { ratos Wistar } \\
\text { macho }\end{array}$ & $\begin{array}{l}\text { Os animais foram divididos em } 4 \text { grupos (terapia } \\
\text { a laser, hidrocolóide, laser com hidrocolóide, } \\
\text { controle). Foi realizada a confecção de uma } \\
\text { ferida, sendo retirado um fragmento de } 0,7 \mathrm{~mm} \\
\text { de diâmetro. A dose foi de } 2 \mathrm{~J} / \mathrm{cm}^{2} \text { com aplicação } \\
\text { diária, com comprimento de onda de } 660 \mathrm{~nm} \text { e } \\
40 \mathrm{~mW} \text { de potência. Foi realizado a comparação } \\
\text { entre os grupos para avaliar a cicatrização das } \\
\text { feridas. }\end{array}$ & $\begin{array}{l}\text { Não houve diferença nos } \\
\text { resultados em relação a } \\
\text { cicatrização nos diferentes } \\
\text { grupos. Porém, observou-se que } \\
\text { os animais ficaram mais calmos } \\
\text { no grupo laser, talvez por } \\
\text { diminuição da dor. }\end{array}$ \\
\hline $\begin{array}{l}\text { De Sousa et al. } \\
\qquad(2017)\end{array}$ & Brasil & $\begin{array}{c}\text { Estudo } \\
\text { Experimental, } \mathrm{n}= \\
36 \text { camundongos }\end{array}$ & $\begin{array}{l}\text { Os } 36 \text { animais foram divididos em } 6 \text { grupos. } \\
\text { Induziu-se um lesão na pele com exérese de } 0,8 \\
\text { mm. Os animais foram avaliados } 7 \text { e } 14 \text { dias } \\
\text { após a formação da lesão com objetivo de } \\
\text { verificar qual recurso terapêutico (laserterapia de } \\
\text { baixa intensidade e ultra-som) é mais eficaz no } \\
\text { processo de cicatrização. Foram utilizadas as } \\
\text { doses de } 3 \text { e } 6 \mathrm{~J} / \mathrm{cm}^{2} \text {, com } 904 \mathrm{~nm} \text { de } \\
\text { comprimento de } 10 \mathrm{~mW} \text { de potência. }\end{array}$ & $\begin{array}{l}\text { O ultrassom pulsado apresentou } \\
\text { resultados significantes e mais } \\
\text { efetivos no que diz respeito a } \\
\text { ação anti-inflamatória, melhor } \\
\text { organização e deposição e } \\
\text { colágeno e fibroblastos. O laser } \\
\text { pode ser utilizado nos primeiros } \\
\text { dias do processo inicial de } \\
\text { cicatrização e o ultrassom na } \\
\text { fase final do processo. }\end{array}$ \\
\hline $\begin{array}{l}\text { Nilforoushzadeh, et } \\
\text { al. (2017) }\end{array}$ & Irã & $\begin{array}{c}\text { Série de casos, } \mathrm{n}= \\
10 \text { pacientes }\end{array}$ & $\begin{array}{l}\text { Foram avaliados } 10 \text { pacientes diabéticos com } \\
\text { queimadura grau III. Realizou-se o transplante } \\
\text { autólogo de fibroblastos associado a laserterapia } \\
\text { de baixa intensidade com dose de } 1 \text { e } 6 \mathrm{~J} / \mathrm{cm}^{2} \text { e } \\
\text { comprimento de onda de } 650 \text { e } 808 \mathrm{~nm} \text {. A } \\
\text { potência utilizada foi de } 150 \text { e } 200 \mathrm{~mW} \text {. }\end{array}$ & $\begin{array}{l}\text { A estratégia de utilizar } 1 \mathrm{~J} / \mathrm{cm}^{2} \text { no } \\
\text { comprimento de onda de } 650 \text { no } \\
\text { leito da lesão e de } 6 \mathrm{~J} / \mathrm{cm}^{2} \text { com } \\
\text { comprimento de onda de } 808 \mathrm{~nm} \\
\text { na margem da ferida, foi e } \\
\text { grande valia e apresentou } \\
\text { resultados satisfatórios. A } \\
\text { associação de laserterapia com o } \\
\text { transplante autólogo de } \\
\text { fibroblastos pode ser } \\
\text { considerado um método eficaz } \\
\text { no tratamento de feridas grandes, }\end{array}$ \\
\hline
\end{tabular}




\begin{tabular}{|c|c|c|c|c|}
\hline & & & & $\begin{array}{l}\text { principalmente em pacientes } \\
\text { diabéticos. }\end{array}$ \\
\hline Fioruzi, et al. (2018) & Irã & $\begin{array}{l}\text { Estudo } \\
\text { Experimental, } \mathrm{n}= \\
60 \text { ratos Wistar } \\
\text { machos }\end{array}$ & $\begin{array}{l}\text { Foi induzido hipotireoidismo em } 60 \text { ratos } \\
\text { divididos em } 5 \text { grupos. Após confirmação do } \\
\text { hipotireoidismo, foi realizado ferida incisional } \\
\text { longitudinal nas costelas dos animais. Os } \\
\text { animais pertencentes ao grupo laser foram } \\
\text { receberam } 0,2 \mathrm{~J} / \mathrm{cm}^{2} \text { com comprimento de onda } \\
\text { de } 810 \mathrm{~nm} \text { e potência de } 1,08 \mathrm{~mW} \text { para avaliar o } \\
\text { efeito combinado da terapia a laser e } \\
\text { levotiroxina na cura de feridas em ratos com } \\
\text { hipotireoidismo }\end{array}$ & $\begin{array}{l}\text { A laserterapia a } 810 \mathrm{~nm} \text { de } \\
\text { comprimento de onda, } 0,2 \mathrm{~J} / \mathrm{cm}^{2} \\
\text { combinados com a aplicação de } \\
\text { levotiroxina melhoraram as } \\
\text { propriedades histológicas das } \\
\text { feridas }\end{array}$ \\
\hline Cunha et al, (2019) & Brasil & $\begin{array}{l}\text { Estudo } \\
\text { Experimental, } \mathrm{n}= \\
60 \text { ratos Wistar } \\
\text { machos }\end{array}$ & $\begin{array}{l}\text { Foram realizadas feridas excisionais e tamanho } 1 \\
\mathrm{~cm} \times 1 \mathrm{~cm} \text { em sessenta ratos para avaliar efeitos } \\
\text { de três protocolos de laser de baixa intensidade } \\
\text { no reparo das cicatrizes. Foram utilizados } 4 \text { e } \\
5 \mathrm{~J} / \mathrm{cm}^{2} \text { diariamente com comprimentos de onda } \\
\text { de } 660 \text { e } 780 \mathrm{~nm} \text { com potência de } 40 \mathrm{~mW} \text {. }\end{array}$ & $\begin{array}{l}\text { A laserterapia de baixa } \\
\text { intensidade proporcionou uma } \\
\text { diminuição significativa na } \\
\text { gravidade da resposta } \\
\text { inflamatória, independente do } \\
\text { protocolo. O protocolo de } 4 \mathrm{~J} / \mathrm{cm}^{2} \\
\text { apresentou as maiores taxas de } \\
\text { deposição de colágeno, } \\
\text { sugerindo que a aplicação diária } \\
\text { induziu um efeito de estimulação } \\
\text { mais intensa. }\end{array}$ \\
\hline Taradaj et al. (2018) & Polônia & $\begin{array}{c}\text { Ensaio clínico } \\
67 \text { pacientes }\end{array}$ & $\begin{array}{l}\text { Foi realizada a FBM em todos os pacientes, } \\
\text { utilizando }- \text { se } 4 \mathrm{~J} / \mathrm{cm}^{2} \text { uma vez por dia, por } 5 \\
\text { vezes por semana, durante } 1 \text { mês. Foram } \\
\text { utilizados os comprimentos de onda de } 658,808 \\
\text { e } 940 \text { nm. Com o objetivo de avaliar o efeito da } \\
\text { terapia a laser em diferentes comprimentos de } \\
\text { onda na expressão de fatores de crescimento e } \\
\text { mediadores inflamatórios nos processos de } \\
\text { cicatrização de lesões por pressão }\end{array}$ & $\begin{array}{l}\text { A cura efetivas das lesões por } \\
\text { pressão está associada a } \\
\text { irradiação com o comprimento } \\
\text { de onda de } 658 \mathrm{~nm} \text {. Acredita-se } \\
\text { esse efeito esteja associado à } \\
\text { inibição dos processos } \\
\text { inflamatórios. }\end{array}$ \\
\hline $\begin{array}{l}\text { Kazemikhoo et al. } \\
\text { (2018) }\end{array}$ & Irã & $\begin{array}{l}\text { Ensaio clínico } \\
\text { randomizado } \\
9 \text { pacientes }\end{array}$ & $\begin{array}{l}\text { Foi realizado a FBM em pacientes com } \\
\text { queimadura de pele, todos os dias por } 7 \text { dias com } \\
\text { comprimento de onda } 655 \mathrm{~nm}, 150 \mathrm{~mW} \text { a } 2 \mathrm{~J} / \mathrm{cm}^{2} \\
\text { no leito da lesão e } 6 \mathrm{~J} / \mathrm{cm}^{2} \text { com comprimento de } \\
\text { onda de } 808 \mathrm{~nm} \text { e } 200 \mathrm{~mW} \text { de potência para as } \\
\text { margens. Avaliar a eficácia da laserterapia após } \\
\text { o enxerto de pele para tratamento de } \\
\text { queimaduras }\end{array}$ & 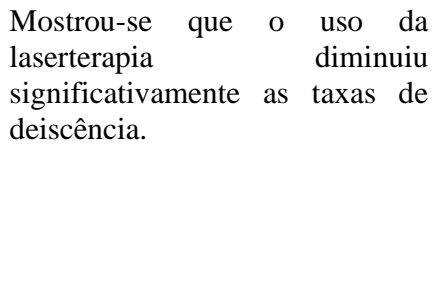 \\
\hline Gomes et al. 2017 & $\begin{array}{l}2017- \\
\text { Brasil }\end{array}$ & $\begin{array}{c}\text { Estudo } \\
\text { Experimental, } \mathrm{n}= \\
12 \text { ratos Wistar } \\
\text { machos }\end{array}$ & $\begin{array}{l}\text { Foram realizadas quatro lesões térmicas por } \\
\text { escaldadura na região dorsal de cada rato. Os } \\
\text { ratos do grupo da laserterapia, receberam a } \\
\text { terapêutica com } 1 \mathrm{~J} / \mathrm{cm}^{2}, 660 \mathrm{~nm} \text { de comprimento } \\
\text { de onda com irradiância de } 6,25 \mathrm{~mW} / \mathrm{cm}^{2} \text { com } \\
160 \text { segundos de exposição por lesão nos dias } 0 \text {, } \\
5 \text { e } 12 \text { pós lesão. O objetivo foi analisar as } \\
\text { características morfológicas e organização das } \\
\text { fibras colágenas dos ratos tratados com } \\
\text { laserterapia e dos ratos com o tratamento padrão } \\
\text {-ouro (sulfadiazina de prata) }\end{array}$ & $\begin{array}{l}\text { Morfologicamente os ratos } \\
\text { tratados com sulfadiazina de } \\
\text { prata e laser foram semelhantes e } \\
\text { ambos proporcionaram um } \\
\text { aumento na organização das } \\
\text { fibras colágenas. Porém, o grupo } \\
\text { de sulfadiazina modulou a } \\
\text { deposição de fibras com mais } \\
\text { eficiência do que o laser. }\end{array}$ \\
\hline
\end{tabular}

Fonte: Autores (2021).

\section{Discussão}

Observou-se nessa revisão uma diferença na densidade de energia utilizada, bem como na frequência de utilização e na potência. Densidades de energia variaram de 1 a $20 \mathrm{~J} / \mathrm{cm}^{2}$, sendo a mais utilizada a de $4 \mathrm{~J} / \mathrm{cm}^{2}$ (Barbosa et al., 2020 ; Filho et al., 2017; Feitosa et al., 2015; Cunha et al., 2019; Taradaj et al. 2018), 5J/cm² (Atasoy et al., 2017; Sousa et al., 2015; Cunha et al., 2019), 6J/ $\mathrm{cm}^{2}$ (De Souza et al., 2018; Nilforoushzadeh et al., 2019; Kasemikhoo et al., 2018), $1 \mathrm{~J} / \mathrm{cm}^{2}$ (Nilforoushzadeh et 
al., 2019; Fioruzi et al., 2018; Gomes et al., 2017), 2J/ $\mathrm{cm}^{2}$ (Martins et al., 2015), 3J/ $\mathrm{cm}^{2}$ (Scherer et al. 2019; De Souza et al.,

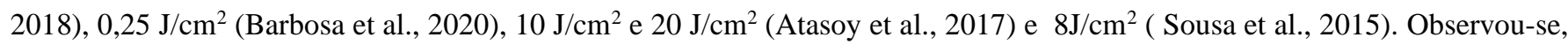
quanto aos parâmetros de aplicação da FBM, que eles variaram de 632,8 a $940 \mathrm{~nm}$, mas a maior parte dos estudos $(12=75 \%)$ utilizou entre 632,8 a $670 \mathrm{~nm}$, demonstrando uma padronização na utilização nas feridas. O número de aplicações variou de 1 a 20 vezes. Outro ponto importante que merece destaque foi a potência utilizada que variou $1,08 \mathrm{~mW}$ a $6 \mathrm{~W}$, porém, a potência de $30 \mathrm{~mW}$ foi a prevalente em seis artigos (37,5\%). Cabe ressaltar que alguns autores utilizaram grupos com diferentes densidades de energia, comprimento de ondas e potência (Barbosa et al., 2020; Atasoy et al., 2017; Sousa et al., 2015; De Sousa et al, 2018; Nilforoushzadeh et al., 2019; Cunha et al., 2019; Taradaj et al., 2018; Kasemikhoo et al., 2018).

Identificou-se que diversos estudos associaram a FBM a outras terapias tópicas utilizadas para cicatrização de feridas (Carvalho et al., 2016; Martins et al., 2015; Nilforoushzadeh et al., 2019; Fioruzi et al., 2018).

Um estudo experimental associou a FBM de $810 \mathrm{~nm}$ de comprimento de onda, com densidade de energia de $0,2 \mathrm{~J} / \mathrm{cm} 2$ com a levotiroxina em 60 ratos com hipotireoidismo divididos em grupos. No grupo de ratos com hipotireoidismo que não recebeu a complementação de levotiroxina, a terapia a laser isolada não apresentou resultados satisfatórios. O hipotireoidismo causa um atraso na cicatrização pois ocorre uma diminuição na organização das fibras colágenas. Quando a levotiroxina foi acrescentada houve uma melhora nas propriedades histológicas da ferida, havendo uma melhora na cicatrização, demonstrando que as condições clínicas do paciente devem ser avaliadas e melhoradas (Fioruzi et al., 2018).

Algumas formas alternativas também são utilizadas para a cicatrização de feridas como a Calêndula officinalis utilizada para o tratamento em úlceras de pé diabéticos. Trata-se de um tipo de Ácidos Graxos Essenciais e foi sugerido com recurso terapêutico, por apresentar ações anti-inflamatórias e cicatrizantes. Essa terapia quando associada a FBM causa melhora da dor, redução da área da ferida, melhora da qualidade de vida e possivelmente diminuição de morbidades no paciente diabético com ferida no pé (Carvalho et al., 2016). Já com o uso de hidrocolóide colocado em ratos com feridas cutâneas, não houve uma diferença de resultados na cicatrização quando associado à FBM. Porém, foi observado que os ratos ficaram mais calmos, provavelmente por diminuição da dor (Martins et al., 2015).

Em estudo experimental utilizando membrana amniótica e FBM com comprimento de onda de $600 \mathrm{~nm}$ e densidade de energia de $4 \mathrm{~J} / \mathrm{cm}^{2}$, observou-se diferentes resultados. O grupo laser que não era associado à membrana amniótica demonstrou um resultado excelente com intensa integração do enxerto a pele, mostrando inclusive na análise microscópica um processo cicatricial mais eficiente. Os grupos que haviam a associação da membrana com a FBM não apresentaram bons resultados, pois parece que a membrana amniótica induziu uma reação inflamatória mais exacerbada (Filho et al., 2017).

Além de terapias tópicas, alguns estudos utilizaram outros equipamentos em consonância com a FBM como gerador de alta frequência (Sousa et al., 2015) e ultrassom (De Sousa et al., 2018) para fins de comparação de efetividade.

$\mathrm{O}$ gerador de alta frequência aplicado com eletrodo de cauterização na faixa máxima de amplitude de 50/60 Hz não apresentou resultados satisfatórios. O grupo que recebeu a FBM com a energia de $5 \mathrm{~J} / \mathrm{cm}^{2}$, apresentou melhores resultados na fase aguda do processo e o grupo que recebeu $8 \mathrm{~J}$ obtiveram os melhores resultados ao fim de 14 dias. O comprimento de onda foi de $660 \mathrm{~nm}$ e a potência de $30 \mathrm{~mW}$ em ambos os grupos de FBM (Sousa et al., 2015). Quando utilizado o ultrassom, observou-se que o FBM apresentou uma diferença significativa em relação a quantidade de fibroblastos e resolução de processo inflamatórios nos primeiros sete dias. O ultrassom apresentou os melhores resultados no $14^{\circ}$ dia com melhor organização e deposição das fibras de colágeno e fibroblastos. Os autores sugerem que o FBM deve ser utilizado no início do tratamento para garantir uma fase inflamatória mais eficiente enquanto que o ultrassom deve ser utilizado ao final do processo (De Sousa et al., 2018).

O tratamento das úlceras por pressão, também podem ser favorecidos com o uso de FBM. Diferentes protocolos de tratamento, com comprimentos de ondas distintas $(658 \mathrm{~nm}, 808 \mathrm{~nm}$ e $940 \mathrm{~nm})$ foram testados. Observou-se que comprimento 
de onda de $658 \mathrm{~nm}$ foi o mais eficaz. Acredita-se que esse feito esteja relacionado à inibição dos processos inflamatórios, à estimulação de angiogênese e proliferação de fibroblastos, conforme os achados da concentração de interleucinas, TNF- $\alpha$ e nas atividades de VEGF, TGF $\beta 1$ nas biópsias das feridas (Taradaj et al., 2018).

A FBM demonstrou uma melhora em feridas de diversas etiologias, entre elas as queimaduras. O transplante autólogo de fibroblastos associado a laserterapia, apresentou resultados satisfatórios e pode ser considerado um método eficaz no tratamento de feridas extensas, principalmente em pacientes diabéticos. Cabe ressaltar que diferentes estudos foram realizados com diferentes energias e comprimentos de onda no leito da lesão e nas margens. Foi utilizado $1 \mathrm{~J} / \mathrm{cm}^{2} \mathrm{com} 650 \mathrm{~nm}$ no leito da ferida e $6 \mathrm{~J} / \mathrm{cm}^{2}$ à $808 \mathrm{~nm}$ nas margens. As potências utilizadas foram de 150 e $200 \mathrm{~W}$ respectivamente (Nilforoushzadeh et al., 2019). Outro estudo semelhante foi realizado em nove pacientes com queimaduras bilaterais, utilizando-se também o laser vermelho para o leito da ferida e o infravermelho para as margens, com pequena modificação da energia, ficando $2 \mathrm{~J}$ com 650 $\mathrm{nm}$ no leito e $6 \mathrm{~J} / \mathrm{cm}^{2}$ com $808 \mathrm{~nm}$ nas margens, com potência de $150 \mathrm{~mW}$ (Kasemikhoo et al., 2018).

Porém, outro estudo apresentou dados divergentes quando utilizou-se a sulfadiazina de prata, considerada padrão ouro no tratamento de queimaduras. O grupo que utilizou a sulfadiazina isolada apresentou modulação da deposição das fibras de colágeno com mais eficiência do que o grupo laser (Gomes et al., 2017).

Um efeito deletério foi comprovado por estudo realizado para investigar a ação da FBM com comprimento de onda de $940 \mathrm{~nm}$ com diversas intensidades de energia na cicatrização óssea. Nesse estudo foram utilizadas as densidades de energia de 5,10 e $20 \mathrm{~J} / \mathrm{cm}^{2}$ com um tempo de irradiação de 10 segundos, porém com potências de $1,5 \mathrm{~W}, 3 \mathrm{~W}$, e $6 \mathrm{~W}$ respectivamente. Não foram encontradas diferenças significativas, no processo cicatricial no que se refere a produção/ inibição de osteócitos, osteoclastos e osteoblastos podendo estar relacionado a alta potência utilizada (Atasoy et al., 2017).

O comprimento de onda de $658 \mathrm{~nm}$ mostrou-se eficaz em estudo que tinha como objetivo investigar os efeitos da fotobiomodulação com comprimento de onda de 658, 830 e 904 nm no reparo de lesões cutâneas em modelo animal. Mostrou um aumento na celularidade, da neovascularização e nas fibras de colágeno tipo III em comparação com outros grupos. Os grupos com comprimento de onda maiores, apresentaram resultados semelhantes ao grupo controle que não havia recebido a FBM (Barbosa et al., 2020).

Também houve melhoras quando utilizado um protocolo com energia de $4 \mathrm{~J} / \mathrm{cm}^{2}$ por ponto com potência de $40 \mathrm{~mW}$ e comprimento de onda de $660 \mathrm{~nm}$ em feridas de ratos Wistar. Observou-se um aumento das taxas de colágeno e a substituição precoce das moléculas de colágeno tipo III por tipo I, proporcionado maior resistência ao tecido reparado quando comparado com outros grupos (Cunha et al., 2019).

Alguns fatores podem interferir no processo cicatricial, entre eles, o tabagismo. O tabaco causa vasoconstrição e diminui a perfusão tecidual, altera a homeostase celular, induzindo um estado pró - inflamatório, o que interfere no processo cicatricial. A enzima mieloperoxidase (MPO) está presente nos leucócitos e causa um desequilíbrio do sistema antioxidante favorecendo danos às células. A MPO é maior em fumantes. A FBM mostrou-se eficaz na diminuição da MPO quando aplicado à uma lesão de tendão calcâneo durante a fase inflamatória aguda, mostrando-se com potencial efeito terapêutico nesse tipo de lesão (Sherer et al., 2019).

Seguindo essa temática, comorbidades como o diabetes também pode ser considerado um fator importante na dificuldade de cicatrização. O FBM utilizado em feridas em pacientes diabéticos, proporciona a diminuição da dor, diminuição do tamanho da ferida, estímulo de neovascularização mais eficiente e aceleração da proliferação celular (Carvalho et al., 2016).

Está cada vez mais claro que a FBM tem alcançado resultados promissores na cicatrização de feridas em pacientes diabéticos, observando-se uma melhora na qualidade de vida por meio da diminuição da dor, melhora no reparo tecidual e diminuição do tamanho das feridas (Carvalho et al., 2016; Sousa et al., 2015; Feitosa et al., 2015). 
Há muitas controversas quanto aos parâmetros que devem ser utilizados para cicatrização das feridas. Isso deve-se aos diversos modelos de estudos utilizados, bem como da falta de padronização das amostras, de parâmetros e período de utilização da FBM.

A cicatrização de feridas ainda pode ser considerada um desafio pela equipe de saúde. A inclusão de novas tecnologias como a FBM em associação às terapias tópicas já existentes, podem auxiliar no tratamento das feridas, trazendo uma mudança de paradigma beneficiando a qualidade de vida do paciente. Sendo assim, esta revisão integrativa pode contribuir para o desenvolvimento de pesquisas futuras e a formulação de protocolos de cicatrização.

\section{Conclusão}

Observou-se que a densidade de energia de $4 \mathrm{~J} / \mathrm{cm}^{2}$ associada ao comprimento de onda vermelho de $658 \mathrm{~nm}$ apresentou maiores resultados na fase aguda da lesão, enquanto energias maiores apresentam maior eficácia no final do processo inflamatório. Associações de produtos tópicos foram realizadas com a FBM, porém somente a Calêndula officinalis apresentou resultados satisfatórios.

Ainda não existe um protocolo para promover a cicatrização de feridas, porém os efeitos da FBM são dependentes da densidade de energia, comprimento de onda e potência do aparelho. Existe uma divergência de evidências, relacionadas à diversidade dos tratamentos utilizados, à falta de padronização na aplicação da laserterapia, bem como dos diferentes parâmetros utilizados. Há necessidade de novo estudos com finalidade de melhor entendimento e estabelecimento de protocolos.

\section{Referências}

Atasoy, K. T., Korkmaz, Y. T., Odaci, E., \& Hanci, H. (2017). The efficacy of low-level 940 nm laser therapy with different energy intensities on bone healing. Brazilian oral research, 31, e7. https://doi.org/10.1590/1807-3107BOR-2017.vol31.0007

Barbosa, L. S, Parisi, J. R., Viana, L. C., Carneiro, M. B., Silva, J. R. T., Silva, M. L., Novaes, R. D. \& Sousa. L. (2020). The photobiomodulation (658, 830 and 904nm) on wound healing in histomorphometric analysis. Fisioter mov 33: e003318. https://doi.org/10.1590/1980-5918.033.ao18

Beheregaray, W. K., Gianotti, G. C., Oliveira, F., Terraciano, P., Bianchi, S., Vidor, C. F., Marcolan, E. A., Contesini, E. O. \& Cirne, L. (2017). Mesenchymal stem cells applied to the inflammatory and proliferative phases of wound healing Arq bras med vet zootec, 69(6), 1591-600. https://doi.org/10.1590/16784162-9461

Carvalho, A. F., Feitosa, M. C., Coelho, N. P., Rebêlo, V. C., Castro, J. G., Sousa, P. R., Feitosa, V. C., \& Arisawa, E. A. (2016). Low-level laser therapy and Calendula officinalis in repairing diabetic foot ulcers. Revista da Escola de Enfermagem da U S P, 50(4), 628-634. https://doi.org/10.1590/S0080623420160000500013

Chung, H., Dai, T., Sharma, S. K., Huang, Y. Y., Carroll, J. D., \& Hamblin, M. R. (2012). The nuts and bolts of low-level laser (light) therapy. Annals of biomedical engineering, 40(2), 516-533. https://doi.org/10.1007/s10439-011-0454-7

Chamusca, F. V., Reis, S. R. A., Lemaire, D. \& Medrado, A. P. (2012). Mediators of the systemic effects of inflammation and photobiomodulatory therapies: a literature review. $R$. Ci. Med. Biol, 11(1), 70-78. http://dx.doi.org/10.9771/cmbio.v11i1.5705

Cunha, J., Carvalho, F., Pereira Filho, R. N., Ribeiro, M., \& de Albuquerque-Júnior, R. (2019). Effects of Different Protocols of Low-Level Laser Therapy on Collagen Deposition in Wound Healing. Brazilian dental journal, 30(4), 317-324. https://doi.org/10.1590/0103-6440201902400

Da Costa Santos, C. M., de Mattos Pimenta, C. A., \& Nobre, M. R. (2007). The PICO strategy for the research question construction and evidence search. Revista latino-americana de enfermagem, 15(3), 508-511. https://doi.org/10.1590/s0104-11692007000300023

De Sousa, A., da Rocha, Í., de Carvalho, A., de Freitas Coelho, N., Feitosa, M., Barros, E., Arisawa, E., \& de Amorim, M. (2018). Comparative Study Between Low Level Laser and Therapeutic Ultrasound in Second Intention Ulcers Repair in Mice. Journal of lasers in medical sciences, 9(2), 134-138. https://doi.org/10.15171/jlms.2018.25

Feitosa, M. C., Carvalho, A. F., Feitosa, V. C., Coelho, I. M., Oliveira, R. A., \& Arisawa, E. Â. (2015). Effects of the Low-Level Laser Therapy (LLLT) in the process of healing diabetic foot ulcers. Acta cirurgica brasileira, 30(12), 852-857. https://doi.org/10.1590/S0102-865020150120000010

Firouzi, A., Norozian, M., Amini, A., Abdollahifar, M. A., Abbaszadeh, H. A., \& Fadaei Fathabadi, F. (2018). Combined Effect of Low-Level Laser Treatment and Levothyroxine on Wound Healing in Rats With Hypothyroidism. Journal of lasers in medical sciences, 9(4), 268-273. https://doi.org/10.15171/jlms.2018.48 
Filho, N. R., Ferreira, M., Pascoli, A., Viéra, R., Pazzini, J., Oliveira R, Salardani, P., Silva, L., Rosseto, S., Albernaz, R.O., Vasconcelos, A., Ferraudo, P.C. \& Moraes, A. (2017). Skin graft epithelialization in rabbit fresh wounds treated with amniotic membrane and/or laser therapy. Arq bras med vet zootec, 69(3), 603-612. https://doi.org/10.1590/1678-4162-9337

Ganong L. H. (1987). Integrative reviews of nursing research. Research in nursing \& health, 10(1), 1-11. https://doi.org/10.1002/nur.4770100103

Gomes, C. F. \& Schapochnik, A. (2017). The therapeutic use of low intensity laser (LLLT) in some diseases and its relation to the performance in speech therapy. Distúrb comun, 29(3), 570-578. http://dx.doi.og/10.23925/2176-2724.2017v29i3p570-578

Gomes, M. T., Campos, G. R., Piccolo N., França, C. M., Guedes, G. H., Lopes, F., Renata, A., Belotto, R. A., Pavani, C., Lima, R. N. \& Silva, D. F. T. (2017). Experimental burns: Comparison between silver sulfadiazine and photobiomodulation. Rev Assoc Med Bras, 63(1), 29-34. https://doi.org/10.1590/1806-9282.63.01.29

Kazemikhoo, N., Vaghardoost, R., Dahmardehei, M., Mokmeli, S., Momeni, M., Nilforoushzadeh, M. A., Ansari, F., Razagi, M. R., Razagi, Z., Amirkhani, M. A., \& Masjedi, M. R. (2018). Evaluation of the Effects of Low Level Laser Therapy on the Healing Process After Skin Graft Surgery in Burned Patients (A Randomized Clinical Trial). Journal of lasers in medical sciences, 9(2), 139-143. https://doi.org/10.15171/jlms.2018.26

Lima, N. E. P., Gomes, G. M., Feitosa, A. N. A., Bezerra, A. L. D. \& Sousa, M. N. A.(2018). Laser therapy low intensity in wound care and practice nurses. Rev enferm UFPI. 7(1),50-56. https://doi.org/10.26694/2238-7234.7150-56

Martelli, A., Andrade, T. A. M. de, \& Santos, G. M. T. dos. (2018). Perspectivas na utilização de fitoterápicos na cicatrização tecidual: revisão sistemática. Archives Of Health Investigation, 7(8). https://doi.org/10.21270/archi.v7i8.3047

Martins, S. S., Torres, O. J. M., Santos, O. J., Limeira, F. A. Jr., Sauaia Filho, E. N, Melo, S. P. C., Pinheiro, R. P. S. \& Silva, B. S. (2015). Analysis of the healing process of the wounds occurring in rats using lasertherapy in association with hydrocolloid. Acta. cir. Bras, 30(10), 681-685. https://doi.org/10.1590/S0102-865020150100000005

Medeiros, A. C., \& Dantas-Filho, A. M. (2017). Cicatrização das feridas cirúrgicas. Journal Of Surgical And Clinical Research,7(2), 87-102. https://doi.org/10.20398/jscr.v7i2.11438

Moher, D., Liberati, A., Tetzlaff, J., Altman, D. G., \& PRISMA Group (2009). Preferred reporting items for systematic reviews and meta-analyses: the PRISMA statement. PLoS medicine, 6(7), e1000097. https://doi.org/10.1371/journal.pmed.1000097

Nilforoushzadeh, M. A., Kazemikhoo, N., Mokmeli, S., Zare, S., Dahmardehei, M., Vaghar Doost, R., Momeni, M., \& Ansari, F. (2019). An Open-Label Study of Low-Level Laser Therapy Followed by Autologous Fibroblast Transplantation for Healing Grade 3 Burn Wounds in Diabetic Patients. Journal of lasers in medical sciences, 10 (Suppl 1), S7-S12. https://doi.org/10.15171/jlms.2019.S2

Oliveira, I. V. P. \& Dias, R. V. C. (2012). Cicatrização de feridas: fases e fatores de influência. Acta Veterinaria Brasílica, 6(4), $267-271$. https://doi.org/10.21708/avb.2012.6.4.2959

Scherer, N. H. B., Silva, A. M. V., Gutierres, J., Veloso, C. F., Pinfildi, C. E. \& Gobbato, R. C. (2019). Laser Photobiomodulation in the acute inflammatory response of the calcaneal tendon injury in rats exposed to cigarette smoke. Fisioter Pesqui, 26(2), 164-169. https://doi.org/10.1590/1809$2950 / 18032726022019$

Silva Neto, C. P., Freire Jr, O. (2017). A gift from Apollo: lasers, history, applications. Rev Bras Ens Fis, 39(1), e1502. https://doi.org/10.1590/1806-9126RBEF-2016-0152

Szwed, D. N. \& Santos, V. L. P. (2016). Growth factors involved in skin healing. Cad da Esc de Saúde.1 (15), 7-17. https://portaldeperiodicos.unibra sil.com.br/index.php/cadernossaude/article/view/2450/2020

Souza, M. T., Silva, M. D., \& Carvalho, R. d. (2010). Integrative review: what is it? How to do it? Einstein (Sao Paulo, Brazil), 8(1), 102-106. https://doi.org/10.1590/S1679-45082010RW1134

Tacani, P. M., Tacani, R. E., Machado, A., Montezello, D., Góes, J., Marx, A. G., \& Liebano, R. E. (2018). High-Frequency Generator in Wound Healing of Marjolin's Ulcer After Carcinoma Resection. Advances in wound care, 7(5), 165-170. https://doi.org/10.1089/wound.2017.0757

Taradaj, J., Shay, B., Dymarek, R., Sopel, M., Walewicz, K., Beeckman, D., Schoonhoven, L., Gefen, A., \& Rosińczuk, J. (2018). Effect of laser therapy on expression of angio- and fibrogenic factors, and cytokine concentrations during the healing process of human pressure ulcers. International journal of medical sciences, 15(11), 1105-1112. https://doi.org/10.7150/ijms.25651

Ursi, E. S., \& Gavão, C. M. (2006). Prevenção de lesões de pele no perioperatório: revisão integrativa da literatura [Perioperative prevention of skin injury: an integrative literature review]. Revista latino-americana de enfermagem, 14(1), 124-131. https://doi.org/10.1590/s0104-11692006000100017

Vieira, R. Q., Sanchez, B. C. S., Fernandes, R. P., Dias, T. N., Aquino, U. M. \& Santos, A. E. (2017). First writings on nursing care in wounds and dressings in Brazil (1916-1947). Hist enferm Rev electronica, 8(2), 106-117. http://here.abennacional.org.br/here/v8/n2/a05.pdf 